\title{
Crosstalk characterization of fabrics elaborated with conductive yarns
}

\author{
Raúl Fernández-García ${ }^{1 *}$, Ignacio Gil ${ }^{1}$, Francesc Cano ${ }^{2}$, Ferran Paré $^{3}$ \\ ${ }^{1}$ Department of Electronic Engineering, Universitat Politècnica de Catalunya, Terrassa, Spain \\ ${ }^{2}$ INTEXTER, Universitat Politècnica de Catalunya, Terrassa, Spain \\ ${ }^{3}$ Department of Textile and Paper Engineering. Universitat Politècnica de Catalunya, Terrassa, Spain. \\ *e-mail: raul.fernandez-garcia@upc.edu
}

\begin{abstract}
The electrical characterization of crosstalk of fabrics elaborated with conductive yarns is investigated. The impact of the source, victim and ground traces location is reported. The experimental results show that the crosstalk in fabrics is higher in comparison with other conductive media. Nevertheless, the results show that the standard strategies to reduce the coupling between lines can be used on fabrics. Doing this, fabrics elaborated with conductive yarns can be used as a conductive media for digital buses, which can help to improve the integration of electronic devices in textile.
\end{abstract}

Keywords-e-textile, crosstalk, yarn, fabrics, wearable, wireless sensor networks.

\section{INTRODUCTION}

In the last years a massive increase of the wireless sensor networks (WSN) has been produced in industrial and domestic applications. The WSN consists of small communication motes. These motes contain a sensing part, a microcontroller, some radiofrequency components, an antenna and power supply (typically a battery) [1]. In this context, many researchers have been paying attention to the integration of WSN in healthcare applications to monitor physiological (i.e., pulse oximetry, respiration rate, temperature, etc.) and physical behaviour (i.e., limb movements, posture, and muscular activity) [2]. In order to increase the usability and comfort of WSN on wearable healthcare applications, the integration of WSN motes on textile should be addressed.

Several papers have been published addressing the design of a specific sensor [3]-[5] or a specific antenna [6]-[9] on textile substrate and some others have been devoted to characterize and model the fabrics [10] and demonstrate the functionally of e-textile as an electromagnetic shielding [11]. Most of these research works overlay these electronic devices on the fabrics by means of embroidery textile techniques [12] or printed techniques [13]. However, in most cases, these techniques are neither comfortable nor user friendly. In this sense, the integration of electronic devices on the own fabrics would be a more comfortable solution for wearable applications. Therefore, the evaluation of the electrical properties of these electronic devices should be analyzed.

In healthcare applications, the distance between the sensor and the microcontroller can be longer than tens of centimeters. For instance, in an electrocardiogram application, a cable from the electrode located in the ankle to the microcontroller, typically located in hip, should be used. Therefore, if an e-

This work was supported by the Spanish Government-MINECO under Project TEC2013-41996-R and AGAUR 2014 SGR 375. textile is used as a conductive medium for this purpose, the propagation of the signal should be analysed taking into account its temporal behaviour.The usefulness of fabrics elaborated with conductive yarn as a conductive media of digital signal has been demonstrated experimentally [14]. However, the crosstalk between fabrics elaborated with conductive yarns has not been deeply analyzed. The coupling can be an important parameter to validate the utility of fabrics as a conductive media of comunication buses, such as the interintegrated circuit bus (I2C). In this paper, the crosstalk of fabrics elaborated with conductive yarn is experimetally characterized taking into account the location of the source, ground and victim traces.

The remainder of the paper is organized as follows. Section II describes the fabrics under test as well as the measurement setup. In Section III the experimental results are shown and discussed. Finally, Section IV reports the main conclusions.

\section{MATERIALS AND METHOD}

\section{A. Technical Fabrics under test}

The fabrics have been woven by a Dornier LWV8/J 190 loom used at the weaving workshop of the Textile and Paper Engineering Department of Universitat Politècnica de Catalunya. The loom runs with a Jacquard Stäubli LX 1600 6480-thread electronic machine and it is able to operate at 650 picks/min. Moreover, it has a $170 \mathrm{~cm}$ wide reed with 10 dents $/ \mathrm{cm}$. The loom was previously threaded using a four yarn/dent.

The fabrics have two different parts, i.e., conductive and non-conductive zones. On the one hand, in a non-conductive zone the yarns were used in two different cotton numbers: $30 / 2$ $\mathrm{Ne}$ (warp) and 40/2 Ne (weft). The density was 38.4 yarns/cm and 15 picks/cm., using an irregular twill weave. On the other hand, the conductive zone was produced with conductor weft yarns from Shieldex Ref. 44113 TR Z200 14. It was woved using the same structure of non-conductive zone. Where the weft density was a 56 picks/cm. (Fig. 1).

The fabric under test (FUT) consists of 4 parallel conductive lines. The width and length of conductive lines are 10 and $1620 \mathrm{~mm}$ respectively, the distance between conductive lines is $10 \mathrm{~mm}$. To clarify the several measurement setups, the conductive lines have been named as trace 1 , trace 2 , trace 3 and trace 4. (Fig. 2). 


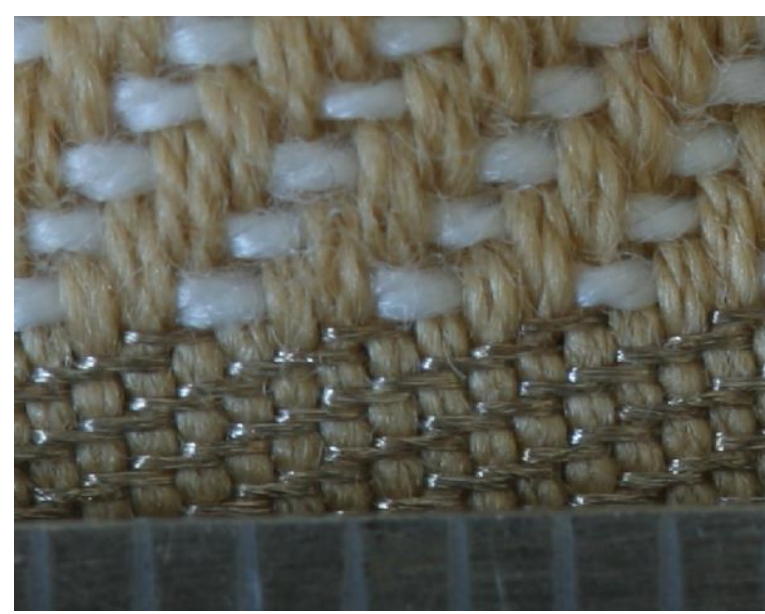

Fig. 1. Close-up image of the joining zone of two traces.

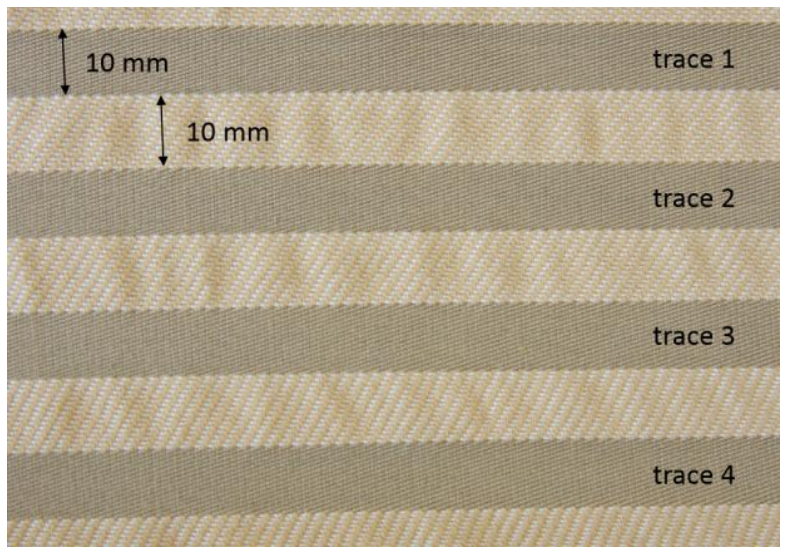

Fig. 2. Fabrics under test.

\section{B. Measurement Setup}

With four parallel lines 24 possible combination of source trace, victim trace and ground trace can be possible. However, due to the FUT symmetry only six different cases have been evaluated. Table 1 summarizes the different cases studied in this work.

Table 1. Summarize of different cases under test.

\begin{tabular}{|c|c|c|c|}
\hline & $\begin{array}{c}\text { Source } \\
\text { trace }\end{array}$ & $\begin{array}{c}\text { Ground } \\
\text { trace }\end{array}$ & $\begin{array}{c}\text { Victim } \\
\text { trace }\end{array}$ \\
\hline Case 1 & 1 & 4 & 2,3 \\
\hline Case 2 & 2 & 4 & 1,3 \\
\hline Case 3 & 3 & 4 & 1,2 \\
\hline Case 4 & 1 & 3 & 2,4 \\
\hline Case 5 & 2 & 3 & 1,4 \\
\hline Case 6 & 4 & 3 & 1,2 \\
\hline
\end{tabular}

The source trace has been connected to a signal generator and a frequency sweep from $100 \mathrm{~Hz}$ to $1 \mathrm{MHz}$ has been done (output impedance $50 \Omega$ ). The victim traces have been permanently connected to Keysight MSO7104A mixed signal oscilloscope by means of Agilent 10073D probe (input impedance of $2.2 \mathrm{M} \Omega$ in parallel with $12 \mathrm{pF}$ ) at both ends. The near end crosstalk (NEXT) and far end crosstalk (FEXT) have been obtained by means of expression 1 :

$$
(\mathrm{N}, \mathrm{F}) \operatorname{EXT}(d B)=20 \log _{10} \frac{V_{\text {victim }(N, F)}}{V_{\text {source }}}
$$

Where $V_{\text {source }}$ is the voltage amplitude of the source and $V_{\text {victim (N.F) }}$ is the voltage amplitude of victim line at the near and far end points. Fig. 3 shows an image of the experimental setup.

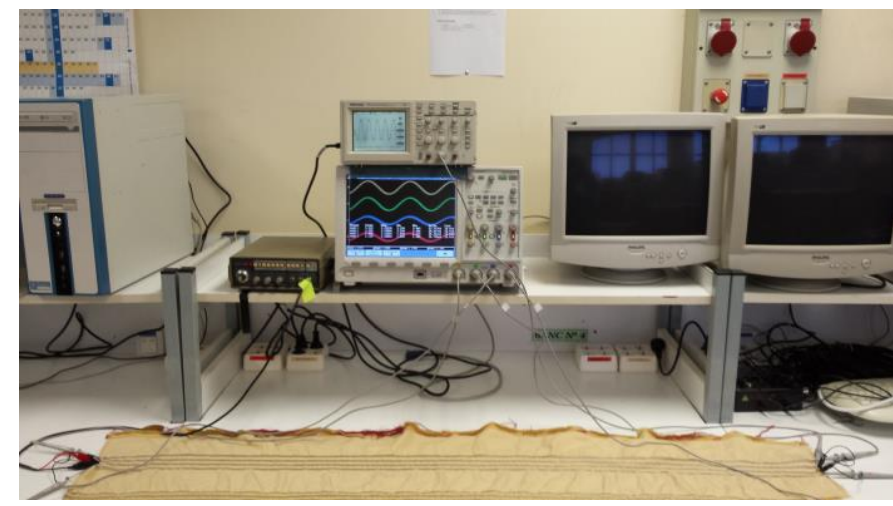

Fig. 3. Experimental setup to measure crosstalk.

\section{RESULTS AND DISCUSSION}

From Fig. 4 to Fig. 9 the NEXT and FEXT crosstalk frequency dependence measurements are shown for the six cases under analysis. In all cases two effects are observed. On the one hand, the crosstalk increases with the frequency up to $10 \mathrm{kHz}$ and remains constant from $10 \mathrm{kHz}$ to $1 \mathrm{MHz}$. On the other hand, no difference between NEXT and FEXT is observed.

The crosstalk frequency dependence is mainly due to the capacitive coupling between adjacent conductive traces. Indeed, below $10 \mathrm{kHz}$ the crosstalk is dominated by the coupling capacitance behaviour, however above $10 \mathrm{kHz}$ the crosstalk remains constant due to the fact that the impedance of the measurement probe is higher than the corresponding to the coupling impedance. The maximum experimental frequency is $1 \mathrm{MHz}$. At this frequency the wavelength is much higher than the fabrics length. Therefore, the FUT can be analysed by means of lumped elements and no difference between NEXT and FEXT is expected. 


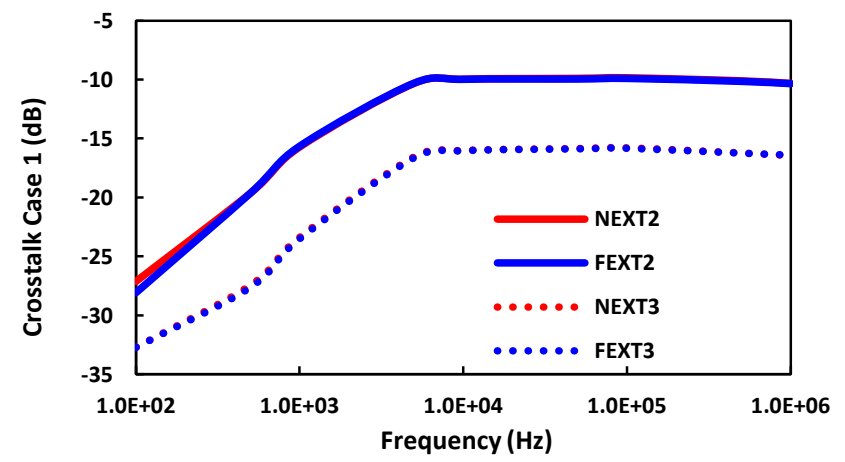

Fig. 4. Case 1 Crosstalk experimental results.

If each case is analysed in detail the next results are observed. In Case 1 , the crosstalk in trace 2 increases from $-28.09 \mathrm{~dB}$ to $-10.35 \mathrm{~dB}$ whereas crosstalk in trace 3 increases from $32.73 \mathrm{~dB}$ up to $-16.41 \mathrm{~dB}$ keeping a difference of about $5 \mathrm{~dB}$ higher in trace 2 with redard to trace 3 , since trace 2 is closer to the source line (trace 1).

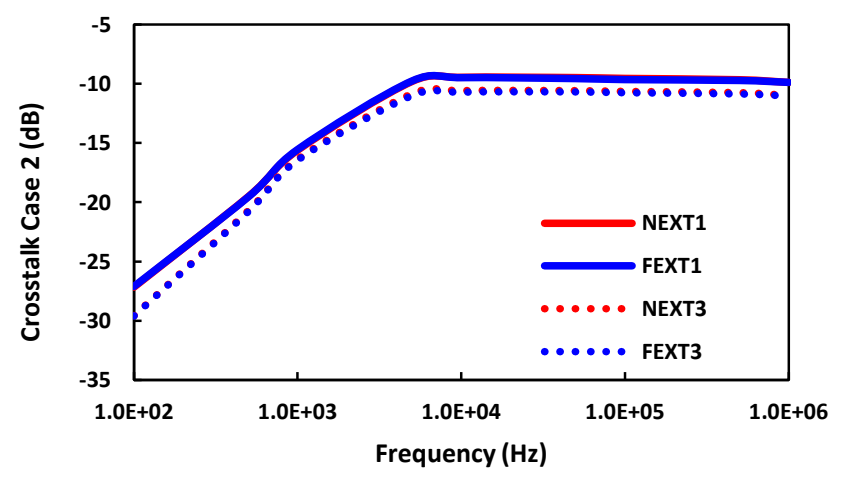

Fig. 5. Case 2 Crosstalk experimental results.

In Case 2, the distance of victim lines (trace1, trace 3 ) to source line (trace 2) is the same. The crosstalk in trace 1 increases from $-27.11 \mathrm{~dB}$ to $-9.89 \mathrm{~dB}$ whereas crosstalk in trace 3 increases from -29.60 up to $-11.02 \mathrm{~dB}$ keeping a maximum difference of about $2.5 \mathrm{~dB}$ at $100 \mathrm{~Hz}$. Although both victim lines show a quite similar behavior, the crosstalk in trace 3 is slightly better due to the fact that trace 3 is closer to the ground line (trace 4).

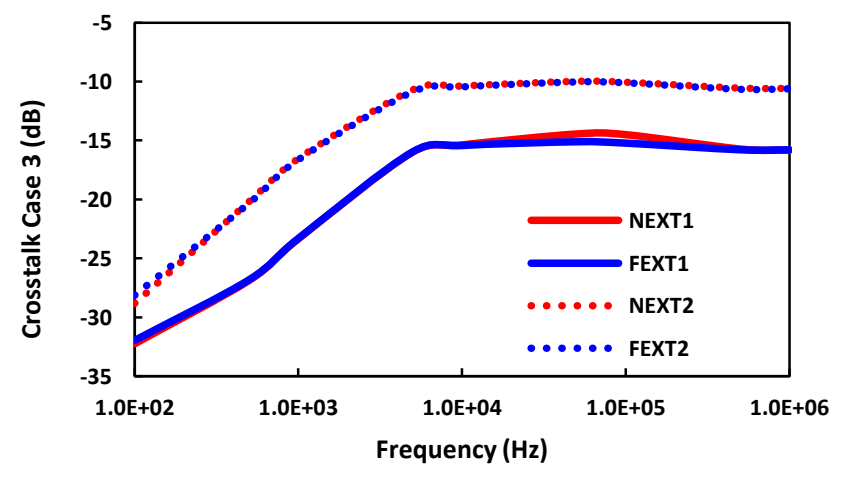

Fig. 6. Case 3 Crosstalk experimental results.

In Case 3, the crosstalk in trace 1 increases from $-31.97 \mathrm{~dB}$ to $15.79 \mathrm{~dB}$ meanwhile crosstalk in trace 2 increases from $28.13 \mathrm{~dB}$ up to $-10.61 \mathrm{~dB}$ by keeping a difference again of about $5 \mathrm{~dB}$ higher in the trace closer to source line.

From Case 4 to Case 6 the ground line consists of the trace 3 and this effect improves the crosstalk behavior in some cases (ground trace behaves like a guard trace). In particular, in case 4 the victim line trace 2 is in the middle of source and ground lines and the crosstalk increases from $-24.76 \mathrm{~dB}$ to $-11.16 \mathrm{~dB}$. However, the crosstalk in victim trace 4 increases from -37.46 $\mathrm{dB}$ to $-23.02 \mathrm{~dB}$ which represents an average improvement of about $12 \mathrm{~dB}$ between both victim traces due to the fact that ground line (trace 3 ) is working such as guard line for trace 4 . The same effect is observed in Case 5 where the crosstalk increases from $-25.30 \mathrm{~dB}$ to $-10.03 \mathrm{~dB}$ for trace 1 and from $36.83 \mathrm{~dB}$ to $-19.77 \mathrm{~dB}$ for trace 4 .

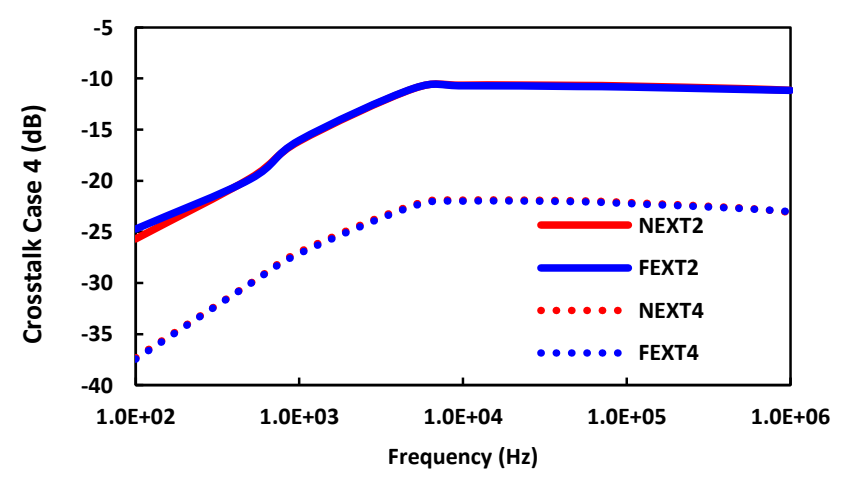

Fig. 7. Case 4 Crosstalk experimental results. 


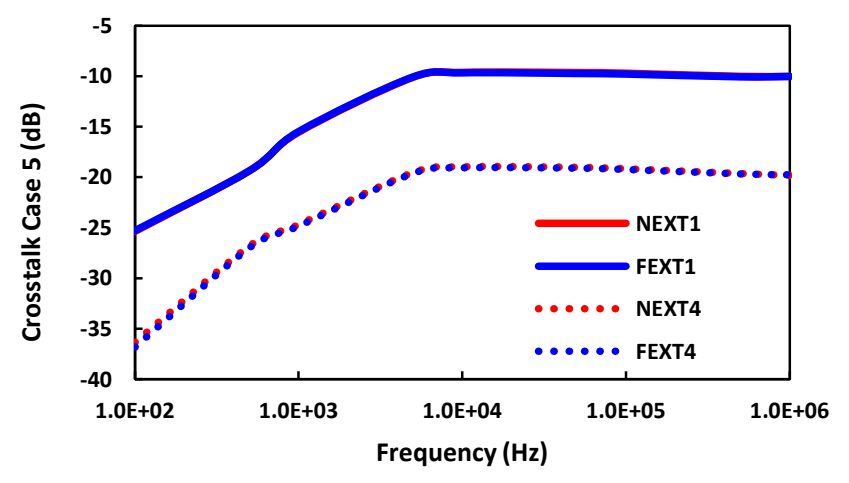

Fig. 8. Case 5 Crosstalk experimental results.

Finally, in Case 6 the ground line (trace 3) is working such as guard line for both victim lines (trace1, trace2). The crosstalk increases from $-34.56 \mathrm{~dB}$ to $-19.78 \mathrm{~dB}$ for trace 2 and from to $-33.23 \mathrm{~dB}$ to $-22.67 \mathrm{~dB}$ for trace 1 . Therefore, Case 6 is the configuration that shows better crosstalk behavior of all. Despite being the better case, it should be noticed that the measured crosstalk values are quite high in comparison with typical coupling levels in standard printed circuit board (PCBs). Therefore, in order to use fabrics elaborated with conductive yarn as propagation media of digital signals, some well-known strategies to improve the crosstalk must be considered. Thus, to increase the distance between source and victim line, to use guard lines, to reduce the length of the traces as much as possible and to use the optimum source and load end impedance will be required techniques to design muti conductor e-textiles

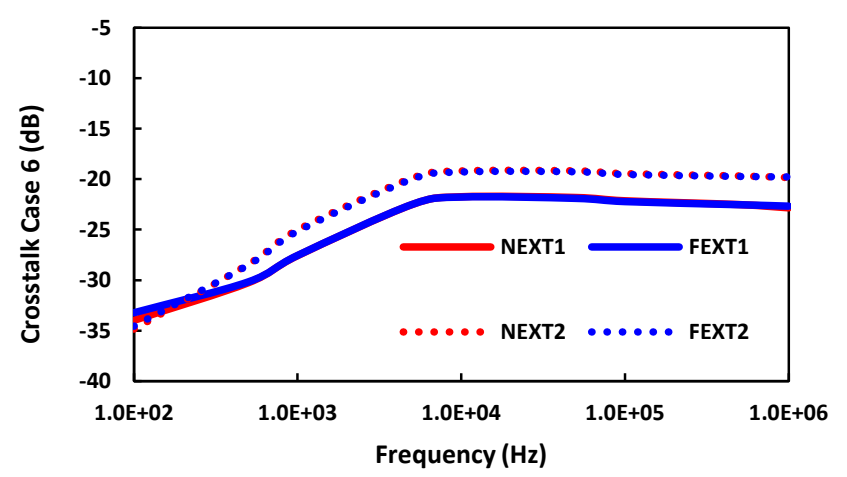

Fig. 9. Case 6 Crosstalk experimental results.

\section{CONCLUSION}

The crosstalk of fabrics elaborated with conductive yarns have been characterized for several cases. The results show that the crosstalk can be a critical parameter that can limit the functinality of fabrics eleborated with conductive yarn as propagation media of digital signals. Therefore, strategies to improve the crosstalk during the design phase should be used. These strategies can guarantee the functionality of fabrics like conductive media of digital buses, helping to increase the usability and comfort of wearable technology.

\section{REFERENCES}

[1] J. Yick, B. Mukherjee, and D. Ghosal, "Wireless sensor network survey," Comput. Networks, vol. 52, no. 12, pp. 2292-2330, Aug. 2008.

[2] J. Ko, C. Lu, M. B. Srivastava, J. A. Stankovic, A. Terzis, and M. Welsh, "Wireless Sensor Networks for Healthcare," Proc. IEEE vol. 98, no. 11, pp. 1947-1960, Nov. 2010.

[3] J.-S. Roh, "Textile touch sensors for wearable and ubiquitous interfaces," Text. Res. J., vol. 84, no. 7, pp. 739-750, Nov. 2013.

[4] L. M. Castano and A. B. Flatau, "Smart fabric sensors and e-textile technologies: a review," Smart Mater. Struct., vol. 23, no. 5, p. 053001, May 2014

[5] G. Cho, K. Jeong, M. J. Paik, Y. Kwun, and M. Sung, "Performance Evaluation of Textile-Based Electrodes and Motion Sensors for Smart Clothing," IEEE Sens. J., vol. 11, no. 12, pp. 3183-3193, Dec. 2011

[6] S. Zhu and R. Langley, "Dual-Band Wearable Textile Antenna on an EBG Substrate," IEEE Trans. Antennas Propag., vol. 57, no. 4 pp. 926-935, Apr. 2009.

[7] S. Choi and S. Lim, "Foldable thin electro-textile antenna array for $4 \times 4$ multiple-input multiple-output mobile router applications," $J$. Electromagn. Waves Appl., vol. 29, no. 3, pp. 375-385, Jan. 2015.

[8] J.-Y. Kim, S.-J. Ha, D. Kim, B. Lee, and C. W. Jung, "Reconfigurable beam steering antenna using U-slot fabric patch for wrist-wearable applications," J. Electromagn. Waves Appl., vol. 26, no. 11-12, pp. 1545-1553, Aug. 2012

[9] T. Kellomäki, J. Virkki, S. Merilampi, and L. Ukkonen, "Towards Washable Wearable Antennas: A Comparison of Coating Materials for Screen-Printed Textile-Based UHF RFID Tags," Int. J. Antennas Propag., vol. 2012, pp. 1-11, Jan. 2012.

[10] J. Lesnikowski and M. Tokarska, "Modeling of selected electric properties of textile signal lines using neural networks," Text. Res. J., vol. 84, no. 3, pp. 290-302, Jul. 2013.

[11] C.-W. Lou, A.-P. Chen, C.-W. Lin, W.-H. Hsing, and J.-H. Lin, "Evaluation on manufacturing technique and electromagnetic shielding effectiveness of functional complex fabrics," $J$ Electromagn. Waves Appl., vol. 28, no. 9, pp. 1031-1043, Apr. 2014

[12] Z. Wang, S. Member, L. Z. Lee, D. Psychoudakis, S. Member, an J. L. Volakis, "Embroidered Multiband Body-Worn Antenna for," IEEE Trans. Antennas Propag., vol. 62, no. 6, pp. 3321-3329, 2014.

[13] Yongsang Kim, Hyejung Kim, and Hoi-Jun Yoo, "Electrical Characterization of Screen-Printed Circuits on the Fabric," IEEE Trans. Adv. Packag., vol. 33, no. 1, pp. 196-205, Feb. 2010.

[14] R. Fernández-García, I. Gil, and F. Cano, "Experimental evaluation of electrical properties of fabrics elaborated with conductive yarns," J. Electromagn. Waves Appl., vol. 30, no. 2, pp. 272-279, Dec 2015 . 